\title{
The neuroanatomical delineation of agentic and affiliative extraversion
}

\author{
Erica N. Grodin • Tara L. White
}

Published online: 25 February 2015

(C) Psychonomic Society, Inc. 2015

\begin{abstract}
Extraversion is a fascinating personality dimension that consists of two major components, agentic extraversion and affiliative extraversion. Agentic extraversion involves incentive motivation and is expressed as a tendency toward assertiveness, persistence, and achievement. Affiliative extraversion involves the positive emotion of social warmth and is expressed as a tendency toward amicability, gregariousness, and affection. Here we investigate the neuroanatomical correlates of the personality traits of agentic and affiliative extraversion using the Multidimensional Personality Questionnaire Brief Form, structural magnetic resonance imaging, and voxel-based morphometry in a sample of 83 healthy adult volunteers. We found that trait agentic extraversion and trait affiliative extraversion were each positively associated with the volume of the medial orbitofrontal cortex bilaterally ( $t^{\prime} \mathrm{s} \geq 2.03, r$ 's $\geq .23, p$ 's $\left.<.05\right)$. Agentic extraversion was specifically and positively related to the volume of the left parahippocampal gyrus $(t=4.08, r=.21, p<$ $.05)$, left cingulate gyrus $(t=4.75, r=.28, p<.05)$, left caudate $(t=4.29, r=.24, p<.05)$, and left precentral gyrus $(t=4.00, r=$ $.18, p<.05)$ in males and females, and the volume of the right nucleus accumbens in males $(t=2.92, r=.20, p<.05)$. Trait affiliative extraversion was not found to be associated with additional regions beyond the medial orbitofrontal cortex. The findings provide the first evidence of a neuroanatomical dissociation between the personality traits of agentic and affiliative extraversion in healthy adults.
\end{abstract}

\footnotetext{
E. N. Grodin · T. L. White $(\triangle)$

Department of Behavioral and Social Sciences, Center for Alcohol and Addiction Studies, Brown University, Box G-S121-4,

121 South Main Street, Providence, RI 02912, USA

e-mail: Tara White@Brown.edu

E. N. Grodin

Brown-NIH Graduate Partnership Program, National Institute on Alcohol Abuse and Alcoholism, Bethesda, MD, USA
}

Keywords Positive emotion · Extraversion · Personality traits $\cdot$ Individual differences $\cdot$ Neuroanatomical basis . Neuroanatomical differences $\cdot$ Neuroanatomical dissociation . Voxel-based morphometry $\cdot$ VBM $\cdot$ MPQ $\cdot$ Healthy adult volunteers $\cdot$ Human subjects $\cdot$ Discriminant validity $\cdot$ Personality neuroscience

Identifying the neural foundations of basic emotional traits is an important goal in affective neuroscience. Extraversion is a particularly fascinating higher-order personality trait that has two major components - agentic and affiliative extraversionthat are separable domains of positive emotionality. Agentic extraversion involves the positive emotions of surgency, ascendency, and ambition, and is behaviorally expressed as a tendency toward assertiveness, persistence, and leadership (Church, 1994; Depue \& Collins, 1999; Patrick, Curtin, \& Tellegen, 2002; Tellegen, 1982). Affiliative extraversion, in contrast, primarily involves the positive emotion of social warmth and is behaviorally expressed as a tendency toward gregariousness and affection (Church, 1994; MorroneStrupinsky \& Lane, 2007; Patrick et al. 2002). The two traits tend to be positively correlated on the order of +.2 to +.3 in most studies (e.g., +.27 in DeYoung, Weisberg, Quilty, \& Peterson, 2013), though a range of larger and smaller correlations have also been observed (e.g., $r=+.43$ and +.52 in DeYoung, Quilty, \& Peterson, 2007; $r=+.14$ in Morrone-Strupinsky \& Lane, 2007, and Watson \& Clark, 1997). The psychometric distinction between the two traits has been well supported by factor-analytic studies. Measures of agentic extraversion load strongly on factors of assertiveness, assuredness, leadership, and social dominance, whereas measures of affiliative extraversion load strongly on a separate factor of enthusiasm and social warmth (Church, 1994; Depue \& Collins, 1999; DeYoung et al. 2007; DeYoung et al. 2013). There are also gender differences in both 
traits. Males as a group tend to report higher scores on measures of trait agentic extraversion than do females, and females tend to report higher scores on measures of trait affiliative extraversion than do males (Helgeson \& Fritz, 1999; Weisberg, DeYoung, \& Hirsh, 2011). Despite this, the distributions of scores on both traits are highly overlapping in males and females, and individual males and females can and do report scores that span the entire range of scores on each trait. Heritability studies indicate strong genetic loadings for both traits, indicating the involvement of biological processes in both personality dimensions (Blonigen, Carlson, Hicks, Krueger, \& Iacono, 2008; Krueger, 2000; Tellegen et al. 1988). These data indicate that trait agentic extraversion and trait affiliative extraversion are phenomenologically and psychometrically distinct, and may involve different brain mechanisms.

The neuroanatomical features that distinguish these traits are not yet understood. A growing number of studies have focused on the structural brain correlates of extraversion, which can be assessed in healthy living humans using voxelbased morphometry (VBM) and tensor-based morphometry (TBM) methods. These techniques use high-resolution anatomical magnetic resonance imaging (MRI) scans to correlate between-subject variation in brain volumes in specific regions with preexisting, between-person variation in one or more independent variables, such as extraversion. Despite an ability to investigate the brain correlates of emotional functioning noninvasively with this approach, such investigations have yielded conflicting findings, with a variety of both positive and negative correlations identified, few of which have been replicated (Coutinho, Sampaio, Ferreira, Soares, \& Goncalves, 2013; Cremers et al. 2011; DeYoung et al. 2010; Forsman, de Manzano, Karabanov, Madison, \& Ullen, 2012; Hu et al. 2011; Lu et al. 2014; Omura, Constable, \& Canli, 2005; Wright et al. 2006). One of the more robust findings has been a positive association between global measures of extraversion and gray matter volume in the medial orbitofrontal cortex (mOFC), a region involved in coding reward, which has been associated with extraversion in three previous studies of healthy adults (Cremers et al. 2011; DeYoung et al. 2010; Omura et al. 2005). Positive correlations have also been identified between extraversion and regional brain volumes in the left amygdala, left fusiform gyrus, left parahippocampal gyrus, right superior temporal gyrus (Omura et al. 2005), and right amygdala (Cremers et al. 2011), which suggests an overall trend toward left-lateralized specialization for extraversion as a higher-order trait. Negative correlations have also been identified, both bilaterally - in the precentral gyrus, superior frontal gyrus, middle frontal gyrus, middle orbitofrontal gyrus, and supramarginal gyrus (Coutinho et al. 2013; Omura et al. 2005) - and unilaterally, in the right hemisphere (superior frontal sulcus, superior frontal gyrus, inferior frontal gyrus, caudate nucleus, angular gyrus, and middle temporal gyrus) as well as the left hemisphere (ventrolateral prefrontal cortex, superior frontal gyrus, intraparietal sulcus, inferior occipital gyrus, and thalamus) (Coutinho et al. 2013; Forsman et al. 2012). The literature is further complicated by several failures to replicate (e.g., no relationship between left amygdala and extraversion (Wright et al. 2006); no relationship between $\mathrm{mOFC}$, amygdala, and extraversion (Koelsch, Skouras, \& Jentschke, 2013); a null finding with regional brain volumes (Hu et al. 2011); and gender differences in the direction of findings in specific regions, such as anterior cingulate (Cremers et al. 2011)). Differences in methods and statistical power have likely contributed, at least in part, to some of these differences between studies. For instance, VBM and TBM differ in their procedures for image registration, spatial normalization, and computation (Ashburner \& Friston, 2000, 2001, 2003; Yanovsky, Leow, Lee, Osher, \& Thompson, 2009), and studies that involve fewer than approximately 80 to 100 participants are underpowered to identify effects that are small in size (Cohen, 1988; DeYoung et al. 2013), increasing the likelihood of Type II error and failures to replicate. The neuroanatomical correlates of extraversion and its subdomains thus remain unclear, despite the importance of this personality domain for subjective well-being, personal achievement, and social bonds.

We propose that some of the variability in the literature could result from a neural heterogeneity among lower-order traits of extraversion. Most VBM and TBM studies conducted to date have used relatively broad, higher-order measures of extraversion, such as the NEO Personality InventoryRevised, to assess this domain. Although these measures capture extraversion at the superfactor level, they typically do not distinguish between the subcomponents of agentic extraversion and affiliative extraversion, which are dissociable subdomains of positive emotionality. Variation in these two traits in different samples could contribute to the differences observed across studies, particularly if agentic extraversion and affiliative extraversion have distinctive profiles of gray matter volumes in healthy adults. One solution to this issue would be to assess agentic extraversion and affiliative extraversion separately, using separate measures of each trait in the same participants. This can be done in healthy volunteers using self-reports on measures such as the Multidimensional Personality Questionnaire, Brief Form (MPQ-BF; Patrick et al. 2002; Tellegen, 1982), an empirically derived personality instrument that assesses agentic extraversion and affiliative extraversion separately, using the primary scales of social potency and social closeness, respectively (Patrick et al. 2002; Tellegen, 1982). Neuroanatomical correlates may then be assessed for each trait using VBM methods, to provide information about the potentially distinct neuroanatomical correlates of each trait. Despite the importance of agentic and affiliative extraversion 
for social and personal well-being throughout the life course, to date only a single study has investigated the brain correlates of the two components of extraversion in the context of normal aging and neurodegenerative disease (Sollberger et al. 2009), and no published data on the structural brain correlates of agentic and affiliative extraversion in healthy adults. The present article begins to fill this gap, with the aim of better understanding the unique neuroanatomical correlates of agentic and affiliative extraversion in healthy adults.

\section{Discrimination of agentic and affiliative extraversion}

We propose that agentic and affiliative extraversion have distinct yet overlapping neuroanatomical correlates in the healthy adult brain. We hypothesize that agentic extraversion should be positively associated with larger gray matter volumes in regions that are innervated by ascending dopamine terminals from the A10 ventral tegmental dopamine cell group, particularly in males who are predisposed to this trait. We hypothesize that affiliative extraversion should be positively associated with larger gray matter volumes in regions innervated by $\beta$-endorphin cells of the hypothalamus, particularly in females who are predisposed to this trait (Depue \& Collins, 1999; Depue \& Morrone-Strupinsky, 2005). These predictions are based on prior work and gender differences in the traits. Agentic extraversion is thought to reflect a behavioral approach system based on positive incentive motivation (Depue, 2006), which brings a person in contact with unconditioned and conditioned positive stimuli and facilitates the frequency and intensity of behaviors oriented toward achieving reward, such as goal-directed action, aggression, and social competition (Depue \& Collins, 1999). Affiliative extraversion, in contrast, is thought to involve individual differences in the mu opiate system and the specific behavioral-motivational processes of (1) social aggregation, in which individuals come together for a social purpose; (2) social consummation, in which individuals engage in social behaviors and experience relaxation and satiety; (3) short-term affective bonding, which is relevant to maternal and paternal parenting behavior and nurturance; and (4) long-term affective bonding, which is relevant to the formation, maintenance, and strength of interpersonal bonds in complex social groups (Depue \& Morrone-Strupinsky, 2005). These behavioral-motivational processes involve both an ability to experience reward relevant to affiliative interactions and an ability to form conditioned preferences for specific individuals based on interpersonal reward (Depue \& Morrone-Strupinsky, 2005). As such, affiliative extraversion should relate to function and possibly structure in regions innervated by dopamine as well as endogenous opiates, which are released during pleasure-inducing behaviors such as sexual activity, birth, lactation and nursing, maternal social interaction, and play
(Machin \& Dunbar, 2011; Nelson \& Panksepp, 1998; Panksepp, 2007; Panksepp, Nelson, \& Siviy, 1994). We thus expected to find a pattern of common and distinct neuroanatomical correlates for the traits of agentic and affiliative extraversion. The brain correlates of these traits may be most observed where biological and cultural processes are relatively aligned and should maximize the developmental expression of preexisting genetic capacities, such that brain correlates of agentic extraversion may be more easily observed in males, and brain correlates of affiliative extraversion may be more easily observed in females. This said, we expect to observe similar directions of the effects in brain substrates in each gender, given the highly overlapping distributions of scores on each trait in males and females.

To address these issues, we investigated the relationship between agentic extraversion, affiliative extraversion, and regional brain volumes in a relatively large sample of healthy adult volunteers $(n=83)$. A sample size of 83 provided $80 \%$ power to detect correlations of $r=.3$, and $99.8 \%$ power to detect correlations of $r=.5$, both evaluated two-tailed at an alpha level of .05 (computations from G*Power 3.1) (Cohen, 1988; Faul, Erdfelder, Buchner, \& Lang, 2009), and it exceeds the mean sample size of previous VBM and TBM studies of extraversion. We assessed agentic extraversion and affiliative extraversion separately, using the MPQ-BF scales of social potency and social closeness, respectively. Scores on each trait were available in all participants, and each participant served as his or her own control. We focused our region-of-interest (ROI) analysis on two brain regions - the orbitofrontal cortex and nucleus accumbens - which we believe could be differentially related to agentic and affiliative extraversion. The orbitofrontal cortex was selected due to its positive relationship with higher-order measures of extraversion in three prior studies of healthy adult volunteers (Cremers et al. 2011; DeYoung et al. 2010; Omura et al. 2005). The nucleus accumbens was selected due to its role in incentive motivation, approach, and goal-directed motor behavior, which is relevant to the agentic extraversion dimension. Agentic extraversion modulates the voluntary approach of salient rewards in the environment (White, Lejuez, $\&$ de Wit, 2007), the magnitude of positive emotion to incentive cues (Morrone, Depue, Scherer, \& White, 2000), and the magnitude of BOLD responses to psychostimulant drug challenge in the right nucleus accumbens. We investigated the mOFC and nucleus accumbens bilaterally, to assess whether a left-hemispheric lateralization would obtain for either trait (for discussion, see Harmon-Jones, Gable, \& Peterson, 2010). Our specific hypotheses were that (1) both agentic extraversion and affiliative extraversion would be positively related to gray matter volume in the mOFC, given its involvement in reward salience and updating the contextual cues of reward, and (2) trait agentic extraversion would be specifically and positively related to gray matter volume in the nucleus accumbens, given the role of this region and trait in incentive motivation and goal-directed 
approach behavior. To protect against false negative (Type II) errors and to investigate relationships in the rest of the brain, we also conducted a whole-brain VBM analysis, which was statistically corrected for the number of comparisons on a voxel-wise basis. Regional brain volumes were thus assessed in the sample using (1) an a-priori ROI analysis, to assess relationships with orbitofrontal cortex and nucleus accumbens, and (2) whole-brain VBM, to assess relationships in the remainder of the brain. Using this approach, we identified a pattern of both common and unique gray matter correlates of agentic extraversion and affiliative extraversion in the healthy adult brain. Our methods and findings are described below.

\section{Method}

\section{Participants}

Participants gave informed consent and underwent structural MRI and personality assessment as a part of one of two smaller studies of the acute effects of alcohol (31 participants) and amphetamine (52 participants). Participants from these smaller studies were combined into a single, larger sample in order to assess the relationship between trait agentic extraversion, trait affiliative extraversion, and brain structure in healthy adult males and females. The participants underwent identical structural imaging procedures and personality assessments, and the structural findings have not been previously reported. Each participant was prescreened using a standardized lab protocol that involved self-report, interview, and medical assessments. The inclusion criteria were age 18-35 years for the amphetamine sample and 21-65 years for the alcohol sample, a minimum high school education, fluency in English, right-handedness, no nonremovable bodily ferromagnetic materials, no history of claustrophobia, no serious medical condition (current or history of cardiac, pulmonary, or liver problems), and for females, no current or intended pregnancy or lactation and using adequate birth control. Individuals with Axis I psychiatric disorders, a history of head trauma with loss of consciousness greater than $10 \mathrm{~min}$, stroke, brain tumor, brain infection, brain surgery, Parkinson's disease, multiple sclerosis, seizure disorder, Huntington's disease, history of mental retardation or developmental disability, and dementia were excluded. Additional exclusion criteria were in effect, to ensure that the participants met NIAAA guidelines for alcohol administration and liver health in the alcohol subsample, and to exclude contraindications for amphetamine administration in the amphetamine subsample. These additional exclusion criteria are not relevant to the present structural data and will not be discussed further. The Brown University Institutional Review Board approved the experimental procedures, with oversight by the Brown University Research Protections Office
(RPO). The research was conducted ethically in accordance with the Helsinki Declaration of 1964 (revised 2013).

\section{Demographics}

Healthy right-handed adults were recruited from Brown University and the surrounding community in Providence, Rhode Island $(N=83)$. The combined sample represented a cross-section of 83 healthy well-educated adults, $18-54$ years of age (mean $=24.9$ years, $S D=7.7$ ), roughly equally distributed by gender (46 female, 37 male), with an average 15 years of education (mean education $=15.1$ years, $S D=1.3$ ) and average body weight (mean $\mathrm{BMI}=23.96, S D=3.3$ ). The sample was racially and ethnically diverse, with $1.2 \%$ of the sample self-identifying as American Indian, $20.5 \%$ as Asian, $12 \%$ as African American, $60.2 \%$ as Caucasian, and $6 \%$ as multiracial/other. The majority of the sample $(91.6 \%)$ self-identified their ethnicity as non-Hispanic, with $8.4 \%$ identifying as Latino/Hispanic.

\section{Personality measures}

The Multidimensional Personality Questionnaire Brief Form (MPQ-BF; Patrick et al. 2002) was used to assess the separate personality traits of agentic extraversion (social potency scale; $\mathrm{SP}$ ) and affiliative extraversion (social closeness scale; SC) in each participant. Personality was assessed at an in-person screening session at the Laboratory of Affective Neuroscience. Participants then reported to the Brown University Magnetic Resonance Facility for structural imaging, which was conducted on a separate day.

Personality scores on the MPQ-BF SP scale (trait agentic extraversion) and $\mathrm{SC}$ scale (trait affiliative extraversion) were in the midrange for scores for each measure (SP mean $=6.62$, $S D=3.23$; $\mathrm{SC}$ mean $=9.19, S D=2.60)$. Scores ranged from the 34 th to the 71st percentile (mean of 54.6th percentile) on SP, and from the 37 th to the 66 th percentile (mean of 54.5 th percentile) on SC, on the basis of national norms available for each trait (Patrick et al. 2002). SP and SC were positively correlated at $+.39, p=.0002$. In males, SP and SC were positively correlated at $+.53, p=.0007$, and in females, $\mathrm{SP}$ and SC were positively correlated at $+.29, p=.05$. Age was not significantly correlated with either trait. Males and females did not differ significantly on their SP or SC scores (male SP mean $=6.8, S D=3.3$, female $\mathrm{SP}$ mean $=6.5, S D=3.2$; male $\mathrm{SC}$ mean $=8.8, S D=2.7$, female $\mathrm{SC}$ mean $=9.5, S D=2.5$ ). The sample thus provides information about gray matter volume correlates of the mid to high range of scores on each trait in both genders.

Image acquisition and analysis

A 3-T Siemens Tim Trio system (Siemens, New York, NY) was used to acquire high-resolution structural images in each 
participant, with whole-brain T1-weighted MPRAGE images acquired in the sagittal plane (resolution $=0.86 \times 0.86 \times$ $0.86 \mathrm{~mm}, \mathrm{TR}=2,250 \mathrm{~ms}, \mathrm{TE}=3.06 \mathrm{~ms}, \mathrm{TI}=900 \mathrm{~ms}$, flip angle $\left.=9^{\circ}, \mathrm{FOV}=220 \mathrm{~mm}\right)$. The structural $\mathrm{T} 1$ images were processed using the VBM toolbox (VBM8; developed by Christian Gaser, University of Jena, Department of Psychiatry) within the SPM8 software package (Wellcome Department of Cognitive Neurology, London, UK).

All images were spatially normalized to standardized anatomical space and segmented into separate images of gray matter, white matter, and cerebrospinal fluid on the basis of a modified Gaussian mixture model (Ashburner \& Friston, 2000). The segmentation procedure was optimized by utilizing the hidden Markov random field (HMRF) based algorithm. This procedure removes isolated voxels of one tissue class that are unlikely to be true members of this tissue type, judging from the tissue class of neighboring voxels. An HMRF weighting of 0.3 was employed. Images were smoothed with a Gaussian kernel of $12 \mathrm{~mm}$ full width at half maximum before the statistical analysis.

A-priori ROI analysis A-priori anatomical ROIs were created for the nucleus accumbens and orbitofrontal cortex bilaterally. These ROIs were created using the WFU PickAtlas (Maldjian, Laurienti, Kraft, \& Burdette, 2003). Age, gender, and the alternate trait were iteratively assessed as nuisance covariates in the regression model, in order to evaluate the association between neuroanatomy and the personality traits of interest, independent of these factors. The full model thus provides information about the unique association between each neuroanatomical region and the personality trait of interest in the sample as a whole (see Table 1), controlling for the contributions of age, gender, and the alternate trait. The interaction of gender and each extraversion trait was analyzed using a full factorial model, which included a gender-by-trait interaction term, correcting for age and the alternate trait as nuisance covariates. The sequentially rejective Holm-Bonferroni method (Holm, 1979) was used to control the overall probability of one or more Type I errors at a fixed family-wise error rate of .05, with two tests for each trait. This approach is conservative, given the likelihood of correlated gray matter volumes in the two a-priori ROIs (see Depue \& Collins, 1999; Depue \& Morrone-Strupinsky, 2005). ROI volumes were extracted from significant clusters using the REX toolbox (http://web.mit.edu/swg/software.htm). Using these extracted clusters, we created scatterplots displaying the correlation between SP or SC scores against the ROI scaled gray matter values. The correlation analysis was conducted using GraphPad Prism 6 software. Significance was evaluated using two-tailed $t$-tests.

Whole-brain VBM analysis The smoothed gray matter images were regressed, using a general linear model, on the trait agentic extraversion and trait affiliative extraversion scores from the MPQ-BF. Age, gender, and the alternate extraversion trait were included as nuisance covariates. The interaction of gender and each extraversion trait was analyzed using a full factorial model, which included a gender-by-trait interaction term, controlling for age and alternate trait as nuisance covariates. For the whole-brain analysis, statistical significance was determined using a voxel height threshold of $p<.05$, corrected for multiple comparisons using the false discovery rate (FDR). Whole-brain results were displayed using the MRIcroGL imaging software (www.mccauslandcenter.sc. edu/mricrogl/).

\section{Results}

Findings from the a-priori and whole-brain VBM analyses are reported in Tables 1, 2, and 3. Both trait agentic extraversion and trait affiliative extraversion were related to volumes in the mOFC. Regional volumes in five additional brain regions were positively associated with trait agentic extraversion. These regions were not significantly associated with trait affiliative extraversion (see Table 3 ). We found no significant interactions between gender and agentic or affiliative extraversion.

Trait agentic extraversion was significantly associated with gray matter volumes in two a-priori regions in both males and females: the right orbitofrontal cortex $(t=3.22, p<.05$

Table 1 A-priori ROI results

\begin{tabular}{|c|c|c|c|c|c|}
\hline Trait and Brain Region & Coordinates (MNI) & Number of Voxels & $t$ Score & $p$ Value Uncorrected & $p$ Value Corrected ${ }^{\dagger}$ \\
\hline \multicolumn{6}{|l|}{ Agentic Extraversion } \\
\hline Right orbitofrontal cortex & $(6,60,-24)$ & 469 & 3.22 & .001 & $<.05$ \\
\hline Left orbitofrontal cortex & $(-15,38,-23)$ & 451 & 2.56 & .006 & $<.05$ \\
\hline \multicolumn{6}{|l|}{ Affiliative Extraversion } \\
\hline Right orbitofrontal cortex & $(48,27,-20)$ & 392 & 3.28 & .001 & $<.05$ \\
\hline Left orbitofrontal cortex & $(-9,39,-27)$ & 76 & 2.03 & .021 & $<.05$ \\
\hline
\end{tabular}

$N=83$. Each $t$ score represents the peak intensity. ${ }^{\dagger}$ The $p$ value is Holm-Bonferroni corrected. 
Table 2 A-priori ROI results by gender

\begin{tabular}{|c|c|c|c|c|c|}
\hline Trait and Brain Region & Coordinates (MNI) & Number of Voxels & $t$ Score & $p$ Value Uncorrected & $p$ Value Corrected ${ }^{\dagger}$ \\
\hline \multicolumn{6}{|l|}{ Agentic Extraversion } \\
\hline \multicolumn{6}{|l|}{ Male } \\
\hline Right orbitofrontal cortex & $(18,48,-12)$ & 381 & 2.92 & .002 & $<.05$ \\
\hline Left orbitofrontal cortex & $(-14,38,-18)$ & 585 & 2.65 & .004 & $<.05$ \\
\hline Right nucleus accumbens ${ }^{*}$ & $(12,8,-8)$ & 80 & 2.92 & .024 & $<.05$ \\
\hline \multicolumn{6}{|l|}{ Female } \\
\hline Right orbitofrontal cortex & $(20,34,-27)$ & 259 & 3.19 & .001 & $<.05$ \\
\hline Left orbitofrontal cortex & $(-15,54,-21)$ & 97 & 2.24 & .015 & $<.05$ \\
\hline \multicolumn{6}{|l|}{ Affiliative Extraversion } \\
\hline \multicolumn{6}{|l|}{ Male } \\
\hline Right orbitofrontal cortex & $(45,26,-18)$ & 1,631 & 5.5 & .0001 & $<.05$ \\
\hline Left orbitofrontal cortex & $(-51,24,-14)$ & 516 & 3.07 & .002 & $<.05$ \\
\hline \multicolumn{6}{|l|}{ Female } \\
\hline Right orbitofrontal cortex & $(12,50,-27)$ & 20 & 1.99 & .023 & $<.05$ \\
\hline Left orbitofrontal cortex & $(-9,42,-27)$ & 22 & 2.06 & .023 & $<.05$ \\
\hline
\end{tabular}

Male $N=37$, Female $N=46$. Each $t$ score represents the peak intensity. ${ }^{\dagger}$ The $p$ value is Holm-Bonferroni corrected. ${ }^{*}$ Regional volume specific to agentic extraversion

Table 3 Whole-brain voxel-based morphometry results

\begin{tabular}{|c|c|c|c|c|c|c|}
\hline Trait and Brain Region & Coordinates (MNI) & Number of Voxels & $t$ Score & $\begin{array}{l}\text { Correlation } \\
\text { Coefficient }(r)\end{array}$ & $\begin{array}{l}p \text { Value } \\
\text { Uncorrected }\end{array}$ & $p$ Value Corrected ${ }^{\dagger}$ \\
\hline \multicolumn{7}{|l|}{ Agentic Extraversion } \\
\hline Left cingulate gyrus ${ }^{*}$ & $(-4.5,-10.5,30)$ & 843 & 4.75 & .28 & .0001 & $<.05$ \\
\hline Left caudate ${ }^{*}$ & $(-21,1.5,18)$ & 154 & 4.29 & .24 & .0001 & $<.05$ \\
\hline Left precentral gyrus ${ }^{*}$ & $(-21,-19.5,64.5)$ & 144 & 4.00 & .18 & .0001 & $<.05$ \\
\hline Left parahippocampal gyrus ${ }^{*}$ & $(-36,-22.5,-19.5)$ & 82 & 4.08 & .21 & .0001 & $<.05$ \\
\hline Right hippocampus & $(27,-30,3)$ & 65 & 3.77 & & .001 & \\
\hline Right superior frontal gyrus & $(3,60,-24)$ & 23 & 3.39 & & .001 & \\
\hline Right fusiform gyrus & $(37.5,-10.52,-27)$ & 7 & 3.26 & & .001 & \\
\hline Right cuneus & $(13.5,-91.5,21)$ & 6 & 3.26 & & .001 & \\
\hline \multicolumn{7}{|c|}{ Agentic Extraversion by Gender Interaction } \\
\hline Left postcentral gyrus & $(-24,-40.5,72)$ & 33 & 4.62 & & .0001 & \\
\hline Left medial frontal gyrus & $(-10.5,34.5,-16.5)$ & 5 & 4.05 & & .0001 & \\
\hline \multicolumn{7}{|l|}{ Affiliative Extraversion } \\
\hline Right cingulate gyrus & $(15,-1.5,42)$ & 87 & 4.11 & & .0001 & \\
\hline Left middle temporal gyrus & $(-52.5,-12,-4.5)$ & 52 & -3.81 & & .001 & \\
\hline Right precentral gyrus & $(46.5,-4.5,48)$ & 42 & 3.47 & & .001 & \\
\hline Left postcentral gyrus & $(-22.5,-40.5,73.5)$ & 36 & -3.94 & & .001 & \\
\hline Right temporal pole & $(46.5,27,-22.5)$ & 28 & 3.57 & & .001 & \\
\hline Right inferior parietal lobule & $(51,-37.5,33)$ & 12 & -3.62 & & .001 & \\
\hline Right medial frontal gyrus & $(3,19.5,48)$ & 11 & 3.40 & & .001 & \\
\hline Right superior parietal lobule & $(33,-78,48)$ & 5 & 3.41 & & .001 & \\
\hline Right middle frontal gyrus & $(28.5,10.5,64.5)$ & 5 & 3.39 & & .001 & \\
\hline
\end{tabular}

$N=83$. Each $t$ score represents the peak intensity. ${ }^{\dagger}$ The $p$ value is corrected for false discovery rate (FDR). ${ }^{*}$ Regional volumes specific to agentic extraversion 
corrected, adjusted alpha $=.025$ ) and left orbitofrontal cortex $(t=2.56, p<.05$ corrected, adjusted alpha $=.025)$. Individual participant scores on trait agentic extraversion were positively correlated with the amount of gray matter measured in each region (right orbitofrontal cortex: $r=.34, p<.05$; left orbitofrontal cortex: $r=.33, p<.05$ ). Trait agentic extraversion was also found to be significantly positively correlated $(r=$ $.20, p<.05)$ with gray matter volume in the right nucleus accumbens in males $(t=2.92, p<.05$ corrected, adjusted alpha $=.05)$. The gender-by-agentic-extraversion interaction term was not statistically significant for any a-priori region. All of the findings with agentic extraversion were positive in direction, which indicates that individuals with higher scores on agentic extraversion had greater gray matter volumes in each ROI. The significant gray matter volume clusters are graphed against SP scores with scatterplots seen in Fig. 1. The whole-brain VBM analysis identified four additional regions that were correlated with trait agentic extraversion: left cingulate gyrus $(t=4.75, r=.28, p<.05$ corrected), left parahippocampal gyrus $(t=4.08, r=.21, p<.05$ corrected $)$, left caudate $(t=4.29, r=.24, p<.05$ corrected $)$, and left precentral gyrus $(t=4.00, r=.18, p<.05$ corrected; see Table 3 and Fig. 2). The associations were positive in direction (Table 3) and were significant after correcting for age, gender, and scores on the alternate trait. Four regions-right hippocampus, right superior frontal gyrus, right cuneus, and right fusiform gyrus - showed positive trends that were not significant after correcting for the number of voxels assessed in the whole-brain analysis and the nuisance covariates $(t ' s \geq 3.26, p$ 's $=.001$ uncorrected; see Table 3$)$. Two regions - the left postcentral gyrus and the left medial frontal gyrus - showed positive trends toward an interaction between gender and trait agentic extraversion, which did not survive FDR correction $\left(t^{\prime} \mathrm{s} \geq 4.05, p^{\prime} \mathrm{s}=.0001\right.$, uncorrected; see Table 3). No regions were negatively correlated with trait agentic extraversion (Tables 1, 2, and 3).

Trait affiliative extraversion was positively correlated with gray matter volumes in the right and left orbitofrontal cortex, a finding similar in direction and magnitude to our findings with agentic extraversion (right mOFC: $t=3.28, p<.05$ corrected, adjusted alpha $=.025$; left mOFC: $t=2.03, p<.05$ corrected, adjusted alpha $=.025)$. The significant gray matter volume clusters are graphed against SC scores. These scatterplots can be seen in Fig. 3. Individual participant scores on trait affiliative extraversion were positively correlated with the amount of gray matter measured in each region (right orbitofrontal cortex: $r=.23, p<.05$; left orbitofrontal cortex: $r=.23, p<.05)$. No significant interactions between gender and trait affiliative extraversion appeared in any a-priori region. The whole-brain VBM analysis identified several positive and negative trends with trait affiliative extraversion, which were not significant after correcting for the number of voxels assessed and nuisance covariates (positive trends: right cingulate gyrus, right precentral gyrus, right temporal pole, right medial frontal gyrus, right superior parietal lobule, and right middle frontal gyrus; negative trends: left middle temporal gyrus, left postcentral gyrus, right inferior parietal lobule; see Table 3). These are illustrated in Fig. 4. We found no significant interactions between gender and trait affiliative extraversion in the whole-brain analysis.

The above findings indicate that trait agentic extraversion and trait affiliative extraversion have overlapping yet distinctive neuroanatomical profiles of gray matter volume in the healthy human brain. Neuroanatomical overlap between agentic and affiliative extraversion was identified in the orbitofrontal cortex, where gray matter volume was strongly positively related to both traits. Trait agentic extraversion had several additional neuroanatomical correlates beyond the orbitofrontal region, with significant findings predominantly localized to the left hemisphere. In contrast, trait affiliative extraversion did not have any neuroanatomical correlates beyond the orbitofrontal region in the same participants. Implications of these findings are discussed below.

\section{Discussion}

To test the neuroanatomical basis of agentic and affiliative extraversion, we evaluated the relationship between trait agentic extraversion, trait affiliative extraversion, and regional gray matter volumes using a-priori ROI and whole-brain VBM methods in a sample of healthy adults. We expected each trait to display a distinctive pattern of regional brain volumes, given the psychometric and phenomenological differences between the two traits. Our findings provide evidence of a clear neuroanatomical dissociation between agentic and affiliative extraversion. Agentic and affiliative extraversion were both positively correlated with gray matter volumes in the mOFC in males and females. Trait agentic extraversion was positively associated with the volume of gray matter in four additional brain areas: left parahippocampal gyrus, left cingulate gyrus, left caudate, and left precentral gyrus (Tables 1 and 3, Figs. 1 and 2) in both males and females. We observed a positive association between gray matter volume in nucleus accumbens and trait agentic extraversion in males (Table 2, Fig. 1). In contrast, trait affiliative extraversion was not significantly related to gray matter volume in regions outside the orbitofrontal cortex. These findings provide the first structural MRI evidence of a neuroanatomical dissociation between the personality traits of agentic and affiliative extraversion in healthy adults. These findings are discussed in turn.

Trait agentic and affiliative extraversion were each associated with the volume of the mOFC. These relationships were positive in direction and significant bilaterally, indicating greater gray matter volumes in individuals with high trait 
a. Left Orbitofrontal Cortex

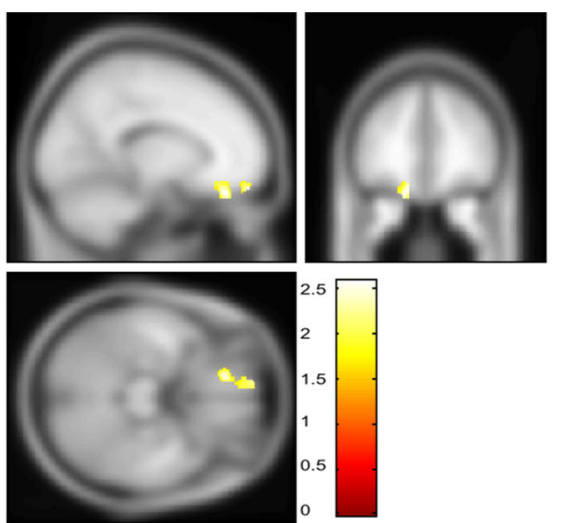

b. Right Orbitofrontal Cortex
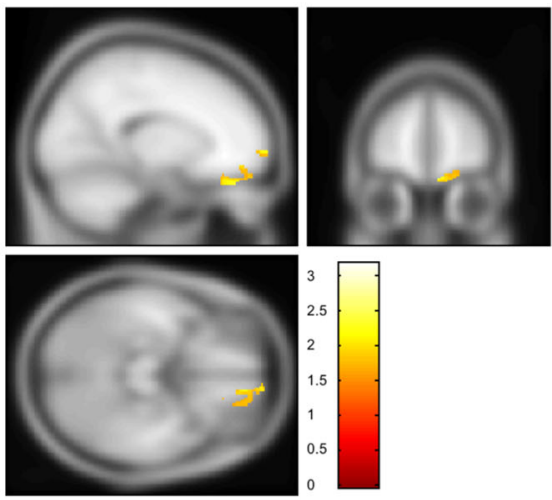

c. Right Nucleus Accumbens - Males

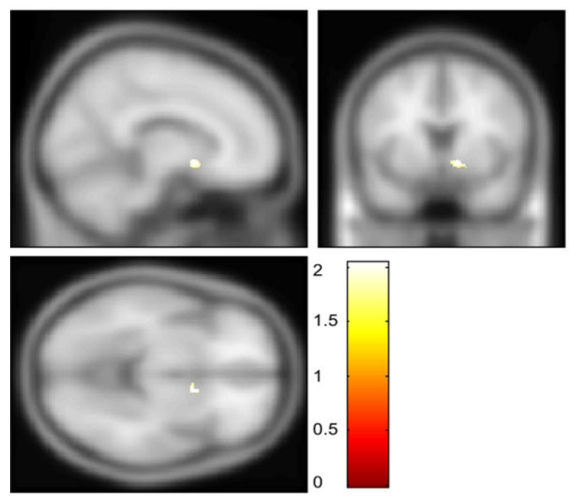

Fig. 1 Social potency (SP) region-of-interest (ROI) results: ROI regions showing significant increases in gray matter in participants with higher SP scores. The left side shows significant gray matter clusters in ROI regions related to social potency. The right side shows scatterplots

scores. This finding indicates a key role of the $\mathrm{mOFC}$ in both affiliative and agentic extraversion in healthy adults. This region has been hypothesized to serve as the primary neural substrate for the overall domain of extraversion (Depue \& Collins, 1999; Goldman-Rakic, Selemon, \& Schwartz, 1984; Miller \& Cohen, 2001), given its role in integrating, maintaining, and updating information relevant to the encoding of reward value, its ability to regulate activity in regions that support
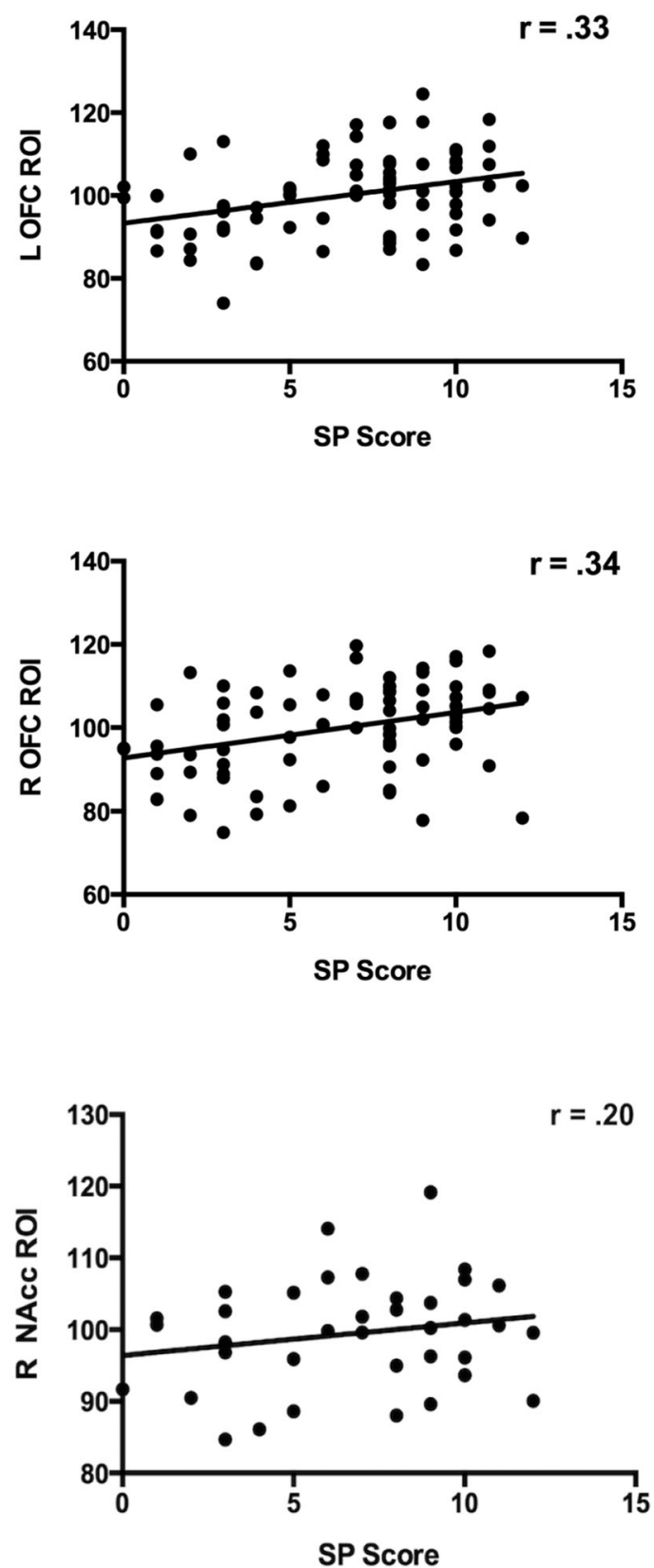

displaying the correlations between the social potency score ( $x$-axis) against the ROI-scaled gray matter values ( $y$-axis). Medial orbitofrontal cortex correlations were significant at $p<.01$, and the right nucleus accumbens correlation was significant at $p<.05$

reward valuation, and its modulation by dopamine projections from the ventral tegmental area (VTA) that facilitate neural integration in these regions (Depue \& Collins, 1999). Structurally, the mOFC is connected with the hippocampus and limbic and paralimbic regions (Depue \& Collins, 1999; Goldman-Rakic et al. 1984; Miller \& Cohen, 2001). These form an interconnected "medial orbitofrontal cortex network," which has been hypothesized to be a neural substrate for extraversion 


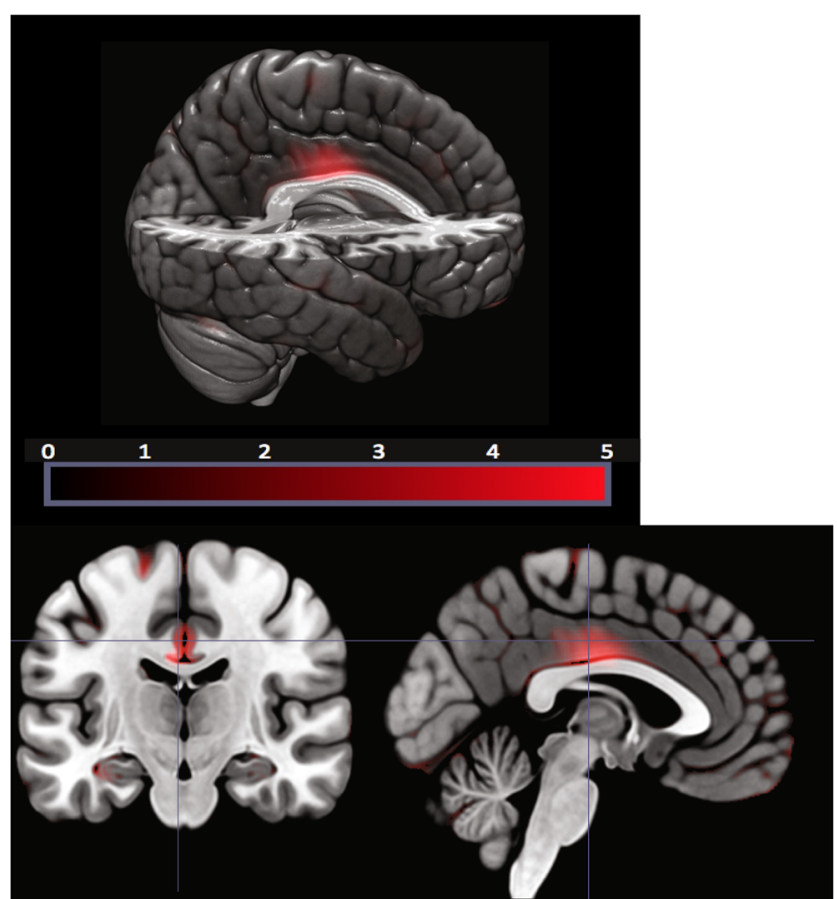

Fig. 2 Social potency (SP) whole brain voxel-based morphometry (VBM) results: Whole brain VBM analysis of gray matter regions associated with social potency. The left parahippocampal gyrus, left cingulate gyrus, left caudate, and left precentral gyrus were significantly correlated with social potency when correcting for multiple comparisons. The colored bar represents $t$-scores from 0 to 5 . See Table 3 for coordinates

(Depue \& Collins, 1999). The present findings support this model and provide evidence that the two major subcomponents of extraversion-agentic and affiliative extraversion - are specifically and independently associated with gray matter volume in the mOFC bilaterally. This may reflect the importance of this area in the evaluation of reward, value-driven choices, and the maintenance of successful choices over time (Noonan, Kolling, Walton, \& Rushworth, 2012), which is relevant to both successful goal-directed behavior (for trait agentic extraversion) and meaningful long-term social bonds (for trait affiliative extraversion). Our findings in $\mathrm{mOFC}$ extend prior findings using more global measures of extraversion in healthy adults (DeYoung et al. 2010; Omura et al. 2005). The above pattern in healthy adults differs from findings in elderly patient populations, which have shown that cortical atrophy in left frontal regions is associated with reductions in agentic interpersonal traits, whereas cortical atrophy in right frontal regions is associated with reductions in affiliative interpersonal traits (Sollberger et al. 2009). The present data indicate that both agentic and affiliative extraversion relate to the volume of gray matter in mOFC bilaterally during healthy young adulthood, when functioning should be optimized.

Agentic extraversion was also specifically associated with gray matter volume in five additional regions: the left parahippocampal gyrus, left cingulate gyrus, left caudate, and left precentral gyrus in both genders (Table 3, Fig. 2), and the right nucleus accumbens in males (Table 2, Fig. 1). These associations were all positive in direction, indicating greater gray matter volumes in individuals predisposed to agentic extraversion. These findings align with existing theories of extraversion. The nucleus accumbens is involved in incentive motivation and is a primary target of ascending dopamine terminals of the A10 dopamine cell group of the ventral tegmentum, which is hypothesized to underlie agentic traits (Depue \& Collins, 1999). The parahippocampus contains D2-receptor-dense regions that innervate dopamine-rich regions of hippocampus (Goldsmith \& Joyce, 1994), creating a coordinated circuit for learning and memory for reward. The positive association between agentic extraversion and gray matter volume in left parahippocampal gyrus could reflect the role of this region in memory encoding and retrieval, responses to reward-related cues (Jasinska, Stein, Kaiser, Naumer, \& Yalachkov, 2014), and navigation of recently learned environments (Boccia, Nemmi, \& Guariglia, 2014), which are relevant to reward-directed behavioral choices. This finding clarifies and extends prior work by Omura and colleagues (2005), who have reported a positive association between the parahippocampal gyrus and a more global measure of extraversion. Three of the regions specifically related to agentic extraversion-the left precentral gyrus, left cingulate gyrus, and left caudate - have not been previously identified as potential neural substrates for extraversion using VBM in healthy volunteers. These regions have, however, been identified in studies using other neuroimaging techniques, such as electroencephalography (EEG) and functional MRI (fMRI), and are consistent with the overall behavioral features of agentic extraversion. Using EEG, Chavanon and colleagues reported a positive association between extraversion and anterior portions of the cingulate (Chavanon, Wacker, \& Stemmler, 2011; Johnson et al. 1999). Using fMRI, Canli and colleagues (2001) reported a positive association between extraversion and BOLD responses in the caudate during the presentation of positive stimuli. The present VBM results thus complement and extend prior findings using EEG and fMRI methods and indicate that gray matter volumes in the left cingulate gyrus, left caudate, and left precentral gyrus are positively associated with trait agentic extraversion in healthy adults. These findings indicate that individuals predisposed to agentic extraversion have larger brain volumes in regions that are involved in the cognitive control of behavior, goal-directed action, the planning and execution of such action, and its positive consequences (Balleine \& O'Doherty, 2010; Chouinard \& Paus, 2006; Deconinck et al. 2014; Grahn, Parkinson, \& Owen, 2008; Hagmann et al. 2008; Hayden \& Platt, 2010; Hollerman, Tremblay, \& Schultz, 1998; Leech, Braga, \& Sharp, 2012), 
a. Left Orbitofrontal Cortex

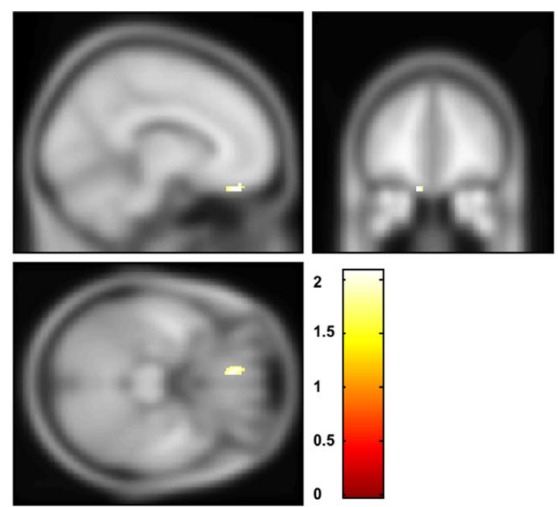

\section{b. Right Orbitofrontal Cortex}
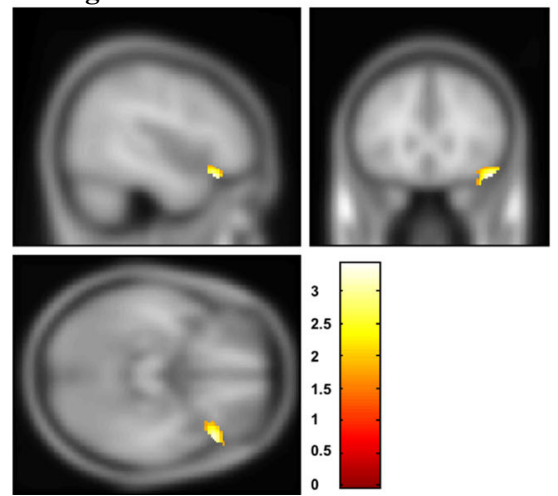

Fig. 3 Social closeness (SC) region of interest (ROI) results: ROI regions showing significant increases in gray matter in participants with higher social closeness scores. The left side shows significant gray matter clusters in ROI regions related to social closeness. The right side shows

which are all consistent with a proclivity toward active approach and goal-directed effort related to this trait. These regions were specifically associated with agentic extraversion, and were unrelated to affiliative extraversion in the same persons (see Table 3).

Agentic extraversion was also positively associated with gray matter volume in the right nucleus accumbens in males (Table 2, Fig. 1). This finding was not due to a gender difference in scores on the trait, as scores on agentic extraversion did not differ between male and female participants. The interaction term between gender and trait agentic extraversion was also not significant, indicating that although effects were observed in males, the findings do not indicate a gender difference in this region. Two prior studies have identified similar relationships between gray matter volumes and extraversion in males. In a sample of adolescents, males exhibited a positive association between trait extraversion and gray matter volume in the left medial frontal gyrus (Blankstein, Chen, Mincic, McGrath, \& Davis, 2009). In a recent sample of adults, males exhibited a positive association between trait extraversion and gray matter volume in the anterior cingulate (Cremers et al. 2011). The present
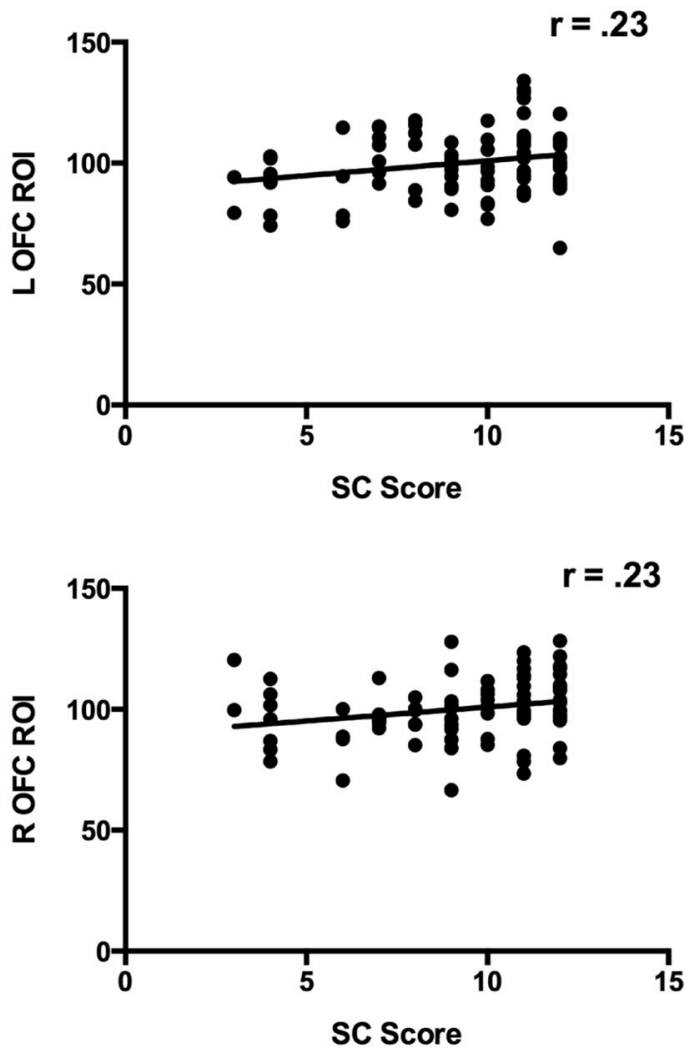

scatterplots displaying the correlation between the social closeness score ( $x$-axis) against the ROI-scaled gray matter values ( $y$-axis). All correlations were significant at $p<0.05$

finding is consistent with these prior VBM findings, as well as with laboratory studies of behavioral responses to psychostimulants. Pharmacologically, administration of standard doses of d-amphetamine, a psychostimulant that alters dopamine levels in the human ventral striatum, has been found to increase the behavioral approach of risky reward in males with high levels of trait agentic extraversion (White et al. 2007). The present finding complements these VBM and laboratory findings and indicates a positive relationship between gray matter volume in nucleus accumbens and agentic extraversion in healthy males.

The above findings are consistent with several neurodevelopmental mechanisms. Agentic extraversion has been hypothesized to relate to dopamine originating from the VTA (Depue \& Collins, 1999). Our findings of greater gray matter volume in $\mathrm{mOFC}$, parahippocampus, cingulate, caudate, and precentral gyrus in individuals with high scores on trait agentic extraversion are consistent with dopamine-facilitated synaptic plasticity in these regions. These regions also support psychological processes that are relevant to the phenomenology of the trait. In adults, the personality trait of agentic extraversion is associated with a sensitivity to rewarding stimuli (Depue \& Collins, 1999; Lucas, Diener, Grob, Suh, \& Shao, 2000), the 


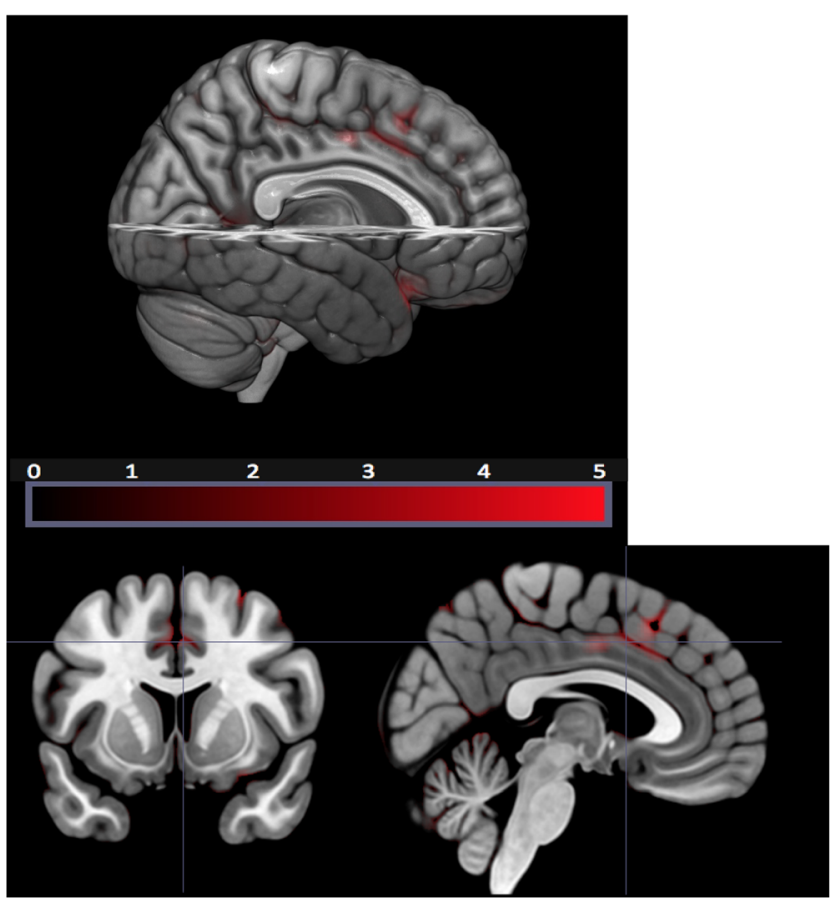

Fig. 4 Social closeness (SC) whole-brain voxel-based morphometry (VBM) results: Whole-brain VBM analysis of gray matter regions correlated with social closeness. No regions survived correction for multiple comparisons. The colored bar represents $t$ scores from 0 to 5 . See Table 3 for more information

selection of action in response to reward (White et al. 2007), vigorous positive emotion in response to positive cues (Morrone et al. 2000), and the binding of rewards, action, and agentic extraversion (Aarts et al. 2012; Andringa, van den Bosch, \& Vlaskamp, 2013). These behavioral, emotional, and cognitive features are consistent with elevated neuronal capacity in the above brain regions.

Affiliative extraversion, in contrast, has been hypothesized to relate to mu opioid mechanisms, in addition to dopaminergic processes (Depue \& Morrone-Strupinsky, 2005). The above differences between agentic extraversion, affiliative extraversion, and gray matter volumes are consistent with a separation in the neural mechanisms for affiliative and agentic extraversion. Of endogenous opiate peptides, $\beta$-endorphin is the most potent and has affinity for the mu opiate receptor (Machin \& Dunbar, 2011). Of the three main classes of opioid receptors (mu, kappa, and delta; Pfeiffer, Pasi, Mehraein, \& Herz, 1982), the mu opioid receptor is believed to be the most likely to mediate opioid effects during social behavior (Depue \& Morrone-Strupinsky, 2005; Stein et al. 2007; Troisi et al. 2011). Regions that receive significant $\beta$-endorphin innervation and/or involve mu-opiate receptor signaling thus represent primary neural targets for trait affiliative extraversion. Relevant to our present findings of elevated gray matter volume in the mOFC with affiliative extraversion, post-mortem studies of the human brain have shown high concentrations of mu opioid receptors in the human orbitofrontal cortex (Machin \& Dunbar, 2011), consistent with opiate contributions to affiliative extraversion. Both sets of findings provide a developmental benchmark from which to better understand the etiology of problems in agentic and affiliative extraversion, such as can occur in normal aging and neurodegenerative disease (see Sollberger et al. 2009).

The present investigation has both strengths and weaknesses. The study's strengths include the use of separate measures of trait agentic extraversion and trait affiliative extraversion in the same individuals, permitting direct comparison of the neuroanatomical correlates for each trait in individuals who serve as their own control; a relatively large sample size for MRI studies, which provides adequate statistical power $(80 \%$ power) to detect effects that are medium in size (i.e., $r$ 's on the order of .3 , per Cohen, 1988, and Faul et al. 2009) and very high power (99.8\% power) to detect relationships that are large in size (i.e., $r$ 's on the order of .5, per Cohen, 1988, and Faul et al. 2009); and empirical evaluation of the structural correlates of agentic and affiliative extraversion using both a-priori ROI and whole-brain VBM methods, which minimize the likelihood of Type I and II errors, respectively. Weaknesses of the study include the use of a cross-sectional design and a correlational data-analytic approach, which prevent conclusions about causality. Our findings may involve three causal pathways, which are not mutually exclusive: (1) Larger gray matter volume in specific regions may support the development of the traits, such that differences in structure contribute to differences in personality; (2) behavioral and emotional components of the traits may facilitate the maintenance of larger gray matter volume in specific brain regions, such that specific behavioral and emotional tendencies may developmentally support the maintenance of gray matter in specific regions; and (3) independent neural processes, such as developmental periods of neuronal blooming and pruning, could contribute to each outcome independently and account for the positive correlations observed here between personality and brain structure in adulthood. The specific neural mechanisms that contribute to our findings are unknown and remain an open question for future work. In addition, our sample size of 83 participants, which is large by the standards of MRI research, is relatively underpowered to detect relationships that may exist but are small in effect size. Evaluation of smaller effects with each trait should be done using larger sample sizes. Toward this end, much larger sample sizes (on the order of 400-800 participants) will be required to achieve $80 \%$ power to detect effects that are small in effect size (e.g., $r$ 's on the order of .10 to .14; Cohen, 1988; Faul et al. 2009). Given the complexity and expense of neuroimaging studies, such sample sizes are generally out of reach for initial proof-of-concept studies such as the present effort. Despite these weaknesses, the present data provide the first neuroanatomical evidence that the personality traits of agentic and affiliative extraversion have distinct yet overlapping patterns of gray matter volume in the healthy human adult brain. 
In summary, the present findings provide evidence of a neuroanatomical distinction between the predispositions toward agentic and affiliative extraversion in healthy adults. Brain volumes that were specifically associated with agentic extraversion include the parahippocampal gyrus, which is involved in learning and memory for reward; the precentral gyrus, cingulate gyrus, and caudate, which are involved in the cognitive control of behavior and the initiation, planning, and execution of voluntary movement toward goals; and (in males) the nucleus accumbens, which is involved in incentive reward (see Tables 1, 2 and 3, Figs. 1 and 2). Agentic extraversion and affiliative extraversion were both associated with the volume of bilateral medial orbitofrontal cortex, which is involved in reward-guided choices and their maintenance (Noonan et al. 2012) and is relevant to goal-directed behavior and long-term social bonding (Table 1). Our findings provide a temporal snapshot of the brain correlates of agentic extraversion and affiliative extraversion in healthy adulthood, which may help us to understand the specific factors involved in the development and maintenance of these important positive emotional traits across the life course.

Author note The authors report that they had no conflicts of interest. This research was supported by grants from the National Institute on Drug Abuse to T.L.W. (Grant Nos. DA R01 020725, DA R21 029189), the Lifespan/Tufts/Brown Center for AIDS Research to T.L.W., the Foundation for Alcohol Research to T.L.W., and the National Institute on Alcohol Abuse and Alcoholism (Grant No. AA P01 019072). We would like to thank Adam Nitenson for his assistance with the figures.

\section{References}

Aarts, H., Bijleveld, E., Custers, R., Dogge, M., Deelder, M., Schutter, D., \& Haren, N. E. (2012). Positive priming and intentional binding: Eye-blink rate predicts reward information effects on the sense of agency. Social Neuroscience, 7, 105-112. doi:10.1080/17470919. 2011.590602

Andringa, T. C., van den Bosch, K. A., \& Vlaskamp, C. (2013). Learning autonomy in two or three steps: Linking open-ended development, authority, and agency to motivation. Frontiers in Psychology, 4, 766. doi:10.3389/fpsyg.2013.00766

Ashburner, J., \& Friston, K. J. (2000). Voxel-based morphometry-The methods. NeuroImage, 11, 805-821. doi:10.1006/nimg.2000.0582

Ashburner, J., \& Friston, K. J. (2001). Why voxel-based morphometry should be used. NeuroImage, 14, 1238-1243. doi:10.1006/nimg. 2001.0961

Ashburner, J., \& Friston, K. J. (2003). Morphometry. In R. S. J. Frackowiak, K. J. Friston, C. Frith, R. Dolan, C. J. Price, S. Zeki, ... W. D. Penny (Eds.), Human brain function (2nd ed.). New York, NY: Academic Press.

Balleine, B. W., \& O'Doherty, J. P. (2010). Human and rodent homologies in action control: Corticostriatal determinants of goal-directed and habitual action. Neuropsychopharmacology, 35, 48-69. doi:10. 1038/npp. 2009.131

Blankstein, U., Chen, J. Y., Mincic, A. M., McGrath, P. A., \& Davis, K. D. (2009). The complex minds of teenagers: neuroanatomy of personality differs between sexes. Neuropsychologia, 47, 599-603. doi: 10.1016/j.neuropsychologia.2008.10.014
Blonigen, D. M., Carlson, M. D., Hicks, B. M., Krueger, R. F., \& Iacono, W. G. (2008). Stability and change in personality traits from late adolescence to early adulthood: A longitudinal twin study. Journal of Personality, 76, 229-266. doi:10.1111/j.14676494.2007.00485.x

Boccia, M., Nemmi, F., \& Guariglia, C. (2014). Neuropsychology of environmental navigation in humans: Review and meta-analysis of FMRI studies in healthy participants. Neuropsychology Review, 24, 236-251. doi:10.1007/s11065-014-9247-8

Canli, T., Zhao, Z., Desmond, J. E., Kang, E., Gross, J., \& Gabrieli, J. D. E. (2001). An fMRI study of personality influences on brain reactivity to emotional stimuli. Behavioral Neuroscience, 115, 33-42. doi:10.1037/0735-7044.115.1.33

Chavanon, M. L., Wacker, J., \& Stemmler, G. (2011). Rostral anterior cingulate activity generates posterior versus anterior theta activity linked to agentic extraversion. Cognitive, Affective, \& Behavioral Neuroscience, 11, 172-185. doi:10.3758/s13415-010-0019-5

Chouinard, P. A., \& Paus, T. (2006). The primary motor and premotor areas of the human cerebral cortex. Neuroscientist, 12, 143-152. doi: $10.1177 / 1073858405284255$

Church, A. T. (1994). Relating the Tellegen and five-factor models of personality structure. Journal of Personality and Social Psychology, 67, 898-909.

Cohen, J. (1988). Stastical power analysis for the behavioral sciences (2nd ed.). Hillsdale, NJ: Erlbaum.

Coutinho, J. F., Sampaio, A., Ferreira, M., Soares, J. M., \& Goncalves, O. F. (2013). Brain correlates of pro-social personality traits: A voxelbased morphometry study. Brain Imaging and Behavior, 7, 293299. doi:10.1007/S11682-013-9227-2

Cremers, H., van Tol, M. J., Roelofs, K., Aleman, A., Zitman, F. G., van Buchem, M. A., \& van der Wee, N. J. A. (2011). Extraversion is linked to volume of the orbitofrontal cortex and amygdala. PLoS ONE, 6, e28421. doi:10.1371/journal.pone.0028421

Deconinck, F.J., Smorenburg, A.R., Benham, A., Ledebt, A., Feltham, M.G., \& Savelsbergh, G.J. (2014). Reflections on mirror therapy: A systematic review of the effect of mirror visual feedback on the brain. Neurorehabilitation \& Neural Repair. doi:10.1177/ 1545968314546134

Depue, R. A. (2006). Interpersonal behavior and the structure of personality: Neurobehavioral foundation of agentic extraversion and affiliation. In T. Canli (Ed.), Biology of personality and individual differences (pp. 60-92). New York, NY: Guilford.

Depue, R. A., \& Collins, P. F. (1999). Neurobiology of the structure of personality: Dopamine, facilitation of incentive motivation, and extraversion. Behavioral and Brain Sciences, 22, 491-517. discussion 518-469.

Depue, R. A., \& Morrone-Strupinsky, J. V. (2005). A neurobehavioral model of affiliative bonding: Implications for conceptualizing a human trait of affiliation. Behavioral and Brain Sciences, 28, 313-350. doi:10.1017/S0140525X05000063. discussion 350-395.

DeYoung, C. G., Hirsh, J. B., Shane, M. S., Papademetris, X., Rajeevan, N., \& Gray, J. R. (2010). Testing predictions from personality neuroscience: Brain structure and the Big Five. Psychological Science, 21, 820-828. doi:10.1177/0956797610370159

DeYoung, C. G., Quilty, L. C., \& Peterson, J. B. (2007). Between facets and domains: 10 aspects of the big five. Journal of Personality and Social Psychology, 93, 880-896. doi:10.1037/0022-3514.93.5.880

DeYoung, C. G., Weisberg, Y. J., Quilty, L. C., \& Peterson, J. B. (2013). Unifying the aspects of the Big Five, the interpersonal circumplex, and trait affiliation. Journal of Personality, 81, 465-475. doi:10. 1111/jopy. 12020

Faul, F., Erdfelder, E., Buchner, A., \& Lang, A. G. (2009). Statistical power analyses using $\mathrm{G}^{*}$ Power 3.1: tests for correlation and regression analyses. Behavior Research Methods, 41, 1149-1160. doi:10. 3758/BRM.41.4.1149 
Forsman, L. J., de Manzano, O., Karabanov, A., Madison, G., \& Ullen, F. (2012). Differences in regional brain volume related to the extraversion-introversion dimension-A voxel based morphometry study. Neuroscience Research, 72, 59-67. doi:10.1016/J.Neures. 2011.10.001

Goldman-Rakic, P. S., Selemon, L. D., \& Schwartz, M. L. (1984). Dual pathways connecting the dorsolateral prefrontal cortex with the hippocampal formation and parahippocampal cortex in the rhesus monkey. Neuroscience, 12, 719-743.

Goldsmith, S. K., \& Joyce, J. N. (1994). Dopamine D2 receptor expression in hippocampus and parahippocampal cortex of rat, cat, and human in relation to tyrosine hydroxylaseimmunoreactive fibers. Hippocampus, 4, 354-373. doi:10. 1002/hipo.450040318

Grahn, J. A., Parkinson, J. A., \& Owen, A. M. (2008). The cognitive functions of the caudate nucleus. Progress in Neurobiology, 86, 141-155. doi:10.1016/j.pneurobio.2008.09.004

Hagmann, P., Cammoun, L., Gigandet, X., Meuli, R., Honey, C. J., Wedeen, V. J., \& Sporns, O. (2008). Mapping the structural core of human cerebral cortex. PLoS Biology, 6, e159. doi:10.1371/ journal.pbio.0060159

Harmon-Jones, E., Gable, P. A., \& Peterson, C. K. (2010). The role of asymmetric frontal cortical activity in emotion-related phenomena: a review and update. Biological Psychology, 84, 451-462. doi:10. 1016/j.biopsycho.2009.08.010

Hayden, B. Y., \& Platt, M. L. (2010). Neurons in anterior cingulate cortex multiplex information about reward and action. Journal of Neuroscience, 30, 3339-3346. doi:10.1523/JNEUROSCI. $4874-$ 09.2010

Helgeson, V. S., \& Fritz, H. L. (1999). Unmitigated agency and unmitigated communion: Distinctions from agency and communion. Journal of Research in Personality, 33, 131-158. doi:10.1006/ Jrpe.1999.2241

Hollerman, J. R., Tremblay, L., \& Schultz, W. (1998). Influence of reward expectation on behavior-related neuronal activity in primate striatum. Journal of Neurophysiology, 80, 947-963.

Holm, S. (1979). A simple sequentially rejective multiple test procedure. Scandinavian Journal of Statistics, 6, 65-70.

Hu, X. C., Erb, M., Ackermann, H., Martin, J. A., Grodd, W., \& Reiterer, S. M. (2011). Voxel-based morphometry studies of personality: Issue of statistical model specification-effect of nuisance covariates. NeuroImage, 54, 1994-2005. doi:10.1016/J. NeuroImage.2010.10.024

Jasinska, A. J., Stein, E. A., Kaiser, J., Naumer, M. J., \& Yalachkov, Y. (2014). Factors modulating neural reactivity to drug cues in addiction: A survey of human neuroimaging studies. Neuroscience \& Biobehavioral Reviews, 38, 1-16. doi:10.1016/j.neubiorev. 2013.10.013

Johnson, D. L., Wiebe, J. S., Gold, S. M., Andreasen, N. C., Hichwa, R. D., Watkins, G. L., \& Boles Ponto, L. L. (1999). Cerebral blood flow and personality: A positron emission tomography study. American Journal of Psychiatry, 156, 252-257.

Koelsch, S., Skouras, S., \& Jentschke, S. (2013). Neural correlates of emotional personality: A structural and functional magnetic resonance imaging study. PLOS ONE, 8, e77196. doi:10.1371/journal. pone.0077196

Krueger, R. F. (2000). Phenotypic, genetic, and nonshared environmental parallels in the structure of personality: A view from the Multidimensional Personality Questionnaire. Journal of Personality and Social Psychology, 79, 1057-1067. doi:10.1037/ 0022-3514.79.6.1057

Leech, R., Braga, R., \& Sharp, D. J. (2012). Echoes of the brain within the posterior cingulate cortex. Journal of Neuroscience, 32, 215-222. doi:10.1523/JNEUROSCI. 3689-11.2012

Lu, F.M., Huo, Y.J., Li, M.L., Chen, H., Liu, F., Wang, Y.F., ... Chen, H. F. (2014). Relationship between personality and gray matter volume in healthy young adults: A voxel-based morphometric study. PLoS ONE, 9. doi:10.1371/journal.pone.0088763

Lucas, R. E., Diener, E., Grob, A., Suh, E. M., \& Shao, L. (2000). Crosscultural evidence for the fundamental features of extraversion. Journal of Personality and Social Psychology, 79, 452-468. doi: 10.1037/0022-3514.79.3.452

Machin, A. J., \& Dunbar, R. I. M. (2011). The brain opioid theory of social attachment: A review of the evidence. Behaviour, 148, 9851025. doi:10.1163/000579511x596624

Maldjian, J. A., Laurienti, P. J., Kraft, R. A., \& Burdette, J. H. (2003). An automated method for neuroanatomic and cytoarchitectonic atlas-based interrogation of fMRI data sets. NeuroImage, 19, 1233-1239.

Miller, E. K., \& Cohen, J. D. (2001). An integrative theory of prefrontal cortex function. Annual Review of Neuroscience, 24, 167-202. doi: 10.1146/annurev.neuro.24.1.167

Morrone, J. V., Depue, R. A., Scherer, A. J., \& White, T. L. (2000). Filminduced incentive motivation and positive activation in relation to agentic and affiliative components of extraversion. Personality and Individual Differences, 29, 199-216. doi:10.1016/S0191-8869(99) 00187-7

Morrone-Strupinsky, J. V., \& Lane, R. D. (2007). Parsing positive emotion in relation to agentic and affiliative components of extraversion. Personality and Individual Differences, 42, 1267-1278. doi:10. 1016/j.paid.2006.10.005

Nelson, E. E., \& Panksepp, J. (1998). Brain substrates of infant-mother attachment: Contributions of opioids, oxytocin, and norepinephrine. Neuroscience \& Biobehavioral Reviews, 22, 437-452. doi:10.1016/ S0149-7634(97)00052-3

Noonan, M. P., Kolling, N., Walton, M. E., \& Rushworth, M. F. (2012). Re-evaluating the role of the orbitofrontal cortex in reward and reinforcement. European Journal of Neuroscience, 35, 997-1010. doi:10.1111/j.1460-9568.2012.08023.x

Omura, K., Constable, R. T., \& Canli, T. (2005). Amygdala gray matter concentration is associated with extraversion and neuroticism. NeuroReport, 16, 1905-1908.

Panksepp, J. (2007). Neuroevolutionary sources of laughter and social joy: Modeling primal human laughter in laboratory rats. Behavioural Brain Research, 182, 231-244. doi:10.1016/j.bbr. 2007.02.015

Panksepp, J., Nelson, E., \& Siviy, S. (1994). Brain opioids and motherinfant social motivation. Acta Paediatrica, 397(Suppl), 40-46.

Patrick, C. J., Curtin, J. J., \& Tellegen, A. (2002). Development and validation of a brief form of the Multidimensional Personality Questionnaire. Psychological Assessment, 14, 150-163. doi:10. 1037/1040-3590.14.2.150

Pfeiffer, A., Pasi, A., Mehraein, P., \& Herz, A. (1982). Opiate receptor binding sites in human brain. Brain Research, 248, 87-96.

Sollberger, M., Stanley, C. M., Wilson, S. M., Gyurak, A., Beckman, V., Growdon, M., \& Rankin, K. P. (2009). Neural basis of interpersonal traits in neurodegenerative diseases. Neuropsychologia, 47, 28122827. doi:10.1016/j.neuropsychologia.2009.06.006

Stein, D. J., van Honk, J., Ipser, J., Solms, M., \& Panksepp, J. (2007). Opioids: From physical pain to the pain of social isolation. CNS Spectrums, 12(669-670), 672-664.

Tellegen, A. (1982). Brief manual for the Multidimensional Personality Questionnaire. Minneapolis, MN: University of Minnesota.

Tellegen, A., Lykken, D. T., Bouchard, T. J., Jr., Wilcox, K. J., Segal, N. L., \& Rich, S. (1988). Personality similarity in twins reared apart and together. Journal of Personality and Social Psychology, 54, 10311039. doi:10.1037/0022-3514.54.6.1031

Troisi, A., Frazzetto, G., Carola, V., Di Lorenzo, G., Coviello, M., D'Amato, F. R., \& Gross, C. (2011). Social hedonic capacity is associated with the $\mathrm{A} 118 \mathrm{G}$ polymorphism of the mu-opioid receptor gene (OPRM1) in adult healthy volunteers and psychiatric patients. 
Social Neuroscience, 6, 88-97. doi:10.1080/17470919.2010. 482786

Watson, D., \& Clark, L. A. (1997). Extraversion and its positive emotional core. In R. Hogan, J. Johnson, \& S. Briggs (Eds.), Handbook of personality psychology (pp. 767-793). New York, NY: Academic Press.

Weisberg, Y. J., DeYoung, C. G., \& Hirsh, J. B. (2011). Gender differences in personality across the ten aspects of the Big Five. Frontiers in Psychology, 2, 178. doi:10.3389/fpsyg.2011.00178

White, T. L., Lejuez, C. W., \& de Wit, H. (2007). Personality and gender differences in effects of $d$-amphetamine on risk taking. Experimental and Clinical Psychopharmacology, 15, 599-609. doi:10.1037/ 1064-1297.15.6.599

Wright, C. I., Williams, D., Feczko, E., Barrett, L. F., Dickerson, B. C., Schwartz, C. E., \& Wedig, M. M. (2006). Neuroanatomical correlates of extraversion and neuroticism. Cerebral Cortex, 16, 18091819. doi:10.1093/Cercor/Bhj118

Yanovsky, I., Leow, A. D., Lee, S., Osher, S. J., \& Thompson, P. M. (2009). Comparing registration methods for mapping brain change using tensor-based morphometry. Medical Image Analysis, 13, 679700. doi:10.1016/j.media.2009.06.002 